\title{
Molecular characterization of breast cancer: a potential novel immune-related IncRNAs signature
}

\author{
Jianguo Lai ${ }^{\dagger}$ (D, Bo Chen ${ }^{\dagger}$, Guochun Zhang, Xuerui Li, Hsiaopei Mok ${ }^{\dagger}$ and Ning Liao ${ }^{*}$
}

\begin{abstract}
Background: Accumulating evidence has demonstrated that immune-related IncRNAs (IRLs) are commonly aberrantly expressed in breast cancer (BC). Thus, we aimed to establish an IRL-based tool to improve prognosis prediction in $B C$ patients.

Methods: We obtained IRL expression profiles in large BC cohorts ( $N=911)$ from The Cancer Genome Atlas (TCGA) database. Then, in light of the correlation between each IRL and recurrence-free survival (RFS), we screened prognostic IRL signatures to construct a novel RFS nomogram via a Cox regression model. Subsequently, the performance of the IRL-based model was evaluated through discrimination, calibration ability, risk stratification ability and decision curve analysis (DCA).

Results: A total of 52 IRLs were obtained from TCGA. Based on multivariate Cox regression analyses, four IRLs (A1BGAS1, AC004477.3, AC004585.1 and AC004854.2) and two risk parameters (tumor subtype and TNM stage) were utilized as independent indicators to develop a novel prognostic model. In terms of predictive accuracy, the IRL-based model was distinctly superior to the TNM staging system (AUC: 0.728 VS $0.673, P=0.010$ ). DCA indicated that our nomogram had favorable clinical practicability. In addition, risk stratification analysis showed that the IRL-based tool efficiently divided BC patients into high- and low-risk groups $(P<0.001)$.
\end{abstract}

Conclusions: A novel IRL-based model was constructed to predict the risk of 5-year RFS in BC. Our model can improve the predictive power of the TNM staging system and identify high-risk patients with tumor recurrence to implement more appropriate treatment strategies.

Keywords: Breast cancer, Immune, IncRNA, Signature, Survival

\section{Background}

Globally, breast cancer (BC) is the most frequently common carcinoma in women $[1,2]$. In light of the statistics of the American Cancer Society, approximately 279,100 new BC cases and 42,690 cancer deaths are estimated

\footnotetext{
*Correspondence: syliaoning@scut.edu.cn

†Jianguo Lai, Bo Chen and Hsiaopei Mok contributed equally to this work. Department of Breast Cancer, Cancer Center, Guangdong Provincial People's Hospital, Guangdong Academy of Medical Sciences, 106 Zhongshan Er Road, Yuexiu district, Guangzhou 510080, Guangdong, China
}

to occur in the United States in 2020 [3]. Although various therapeutic strategies are currently being applied to improve $\mathrm{BC}$ prognosis, including surgery, chemotherapy, radiotherapy, endocrine therapy and targeted therapy, many BC patients still have poor survival outcomes because of recurrence [4]. Survival prognosis prediction is mainly based on the American Joint Committee on Cancer (AJCC) tumor-node-metastasis (TNM) staging system. However, it should be noted that BC patients at the same TNM stage may have distinct survival outcomes [5]. This highlights that the TNM staging system is inadequate to achieve accurate prognosis evaluation and that

(c) The Author(s) 2020. This article is licensed under a Creative Commons Attribution 4.0 International License, which permits use, sharing, adaptation, distribution and reproduction in any medium or format, as long as you give appropriate credit to the original author(s) and the source, provide a link to the Creative Commons licence, and indicate if changes were made. The images or other third party material in this article are included in the article's Creative Commons licence, unless indicated otherwise in a credit line to the material. If material is not included in the article's Creative Commons licence and your intended use is not permitted by statutory regulation or exceeds the permitted use, you will need to obtain permission directly from the copyright holder. To view a copy of this licence, visit http://creativeco mmons.org/licenses/by/4.0/. The Creative Commons Public Domain Dedication waiver (http://creativecommons.org/publicdomain/ zero/1.0/) applies to the data made available in this article, unless otherwise stated in a credit line to the data. 
it is unable to reflect the biological heterogeneity of $\mathrm{BC}$. For example, the system is mainly based on clinicopathological parameters rather than on molecular signatures $[6,7]$. Increasing evidence has revealed that molecular biomarkers have the potential to improve prognostic assessment and identify high-risk cancer patients [8-14]. Accordingly, there is an urgent need to screen effective molecular biomarkers for improving survival prognosis prediction and identify high-risk BC patients with tumor recurrence.

With advancements in transcriptomics, long noncoding RNAs (lncRNAs) have captured considerable attention in human cancers in the past decade [1519]. LncRNAs are a class of mRNA-like transcripts with a length of $>200$ nucleotides that lack proteincoding ability. Accumulating evidence indicates that lncRNAs play important roles in various biological processes, including transcriptional modifications, cell proliferation, differentiation, epigenetic modulation, and immune system-modulated pathways [20-25]. For example, lncRNA SNHG1 enhances the differentiation of Treg cells to provoke immune escape in BC [26]. LncRNA AC025580.2 has a positive influence on immune escape in pancreatic cancer [27]. In addition, a large number of lncRNAs have been confirmed to act as diagnostic and prognostic biomarkers or potential therapeutic targets in multiple tumors [11, 28-32]. However, the role of immunerelated lncRNAs (IRLs) in the prognostic evaluation of $\mathrm{BC}$ remains unclear.

Hence, the objective of this study was to establish and validate a novel IRL model to predict the 5-year RFS of $\mathrm{BC}$ patients. This tool is able to improve the predictive accuracy of the TNM staging system and identify highrisk $B C$ patients with tumor recurrence to facilitate optimal therapeutic schemes.

\section{Methods}

\section{Patients and study design}

Gene and lncRNA expression profiles as well as corresponding $\mathrm{BC}$ cases information were acquired from The Cancer Genome Atlas (TCGA) database. The inclusion criteria were as follows: (1) pathologically confirmed invasive BC, (2) complete follow-up data, including RFS status and survival time, and (3) survival time $>1$ month. The following clinical parameters for each patient were collected: age, estrogen receptor (ER) status, progesterone receptor (PR) status, T stage, N stage, TNM stage, human epithelial growth factor receptor 2 (HER2) status, tumor subtype, RFS, and survival time. The Research Ethics Committee of Guangdong Provincial People's Hospital (GDPH) approved our study. As the study used data from the database TCGA, written informed consent was waived.

\section{Establishment of IRL risk score and the IRL-based model}

First, immune-related genes (IRGs) were retrieved from the Molecular Signatures Database (MSDB) v4.0 (immune response M19817, and immune system process M13664) [33, 34]. According to the GENCODE project, lncRNA expression data were obtained from the gene expression profile [35]. Next, IRLs were defined based on association analysis between the mRNA expression level and lncRNA expression data $(|\mathrm{R}|>0.7, P<0.05)$. Subsequently, univariate Cox proportional hazards regression analysis (CPHRA) was conducted to explore the association between the IRL expression level and patient RFS. Multivariate CPHRA was carried out to investigate independent prognostic IRLs. Regarding multivariate analysis, the collinearity diagnosis was confirmed via the variance inflation factor (VIF), and the final independent prognostic IRLs were selected to develop the IRL-based risk score, as follows: IRL risk score $=$ sum of coefficients $\times$ expression level of IRLs. Finally, a novel IRL-based model incorporating the IRL signature and clinical variables was constructed to improve the prediction power of TNM staging in $\mathrm{BC}$ patients.

\section{Assessment of the IRL-based nomogram}

The area under the curve (AUC) of the time-dependent receiver operating characteristic (ROC) curve for 5-year RFS was calculated to evaluate the performance of the IRL-based model. The nomogram score for each patient was allocated on the basis of the IRL-based nomogram. Patients were divided into low- and high-risk nomogram score groups using the median score as the cutoff point. Subgroup analysis was applied to assess the risk stratification ability of the IRL-based model. A calibration curve was drawn to estimate the calibration ability of the IRLbased nomogram. Decision curve analysis (DCA) was used to weigh the clinical practicability of the IRL-based nomogram [36-41].

\section{Gene enrichment analysis of the IRL signature}

Gene enrichment analysis of the four IRLs was applied using Metascape, a free online method for gene annotation [42]. Functional pathway enrichment was assessed on the basis of co-expressing genes of the four IRLs in the same module.

\section{Statistical analysis}

The $X^{2}$ test, Fisher's exact test or Mann-Whitney U test was employed to explore differences in indicators. The optimal cut-off values of IRL-based nomogram scores were calculated via X-tile software, version 3.6.1 (Yale 
University, New Haven, CT, USA). Kaplan-Meier survival analysis and the log-rank test were used to examine the 5-year RFS difference between low- and high-risk groups. All statistical analyses were utilized via $\mathrm{R}$ software (www.r-project.org, version 3.6.1) and Stata/MP, version 14.0 (StataCorp LP, College Station, TX). A P value $<0.05$ was defined as significant.

\section{Results}

\section{Baseline characteristics}

In total, 911 BC samples were collected as the primary cohort in our study. As in our previous studies, each patient can be assigned a computer-generated allocation number ( 0 or 1$)$ based on Stata software, and two cohorts were differentiated from the primary cohort according to the allocation number. Of the total, 456 patients (allocation number $=0$ ) were considered as the validation cohort $[4,5]$. The baseline characteristics of all patients in the two datasets are shown in Table 1. There were no significant differences between the variables examined in the two independent cohorts (all $P>0.05)$. The median age of the included patients was 58 years (IQR: $48-66)$ and 58 years (IQR: $48-65$ ) in the two datasets.

\section{Identification of the IRL signature}

First, 332 IRGs were collected from Molecular Signatures Database (MSDB), and the expression profiles of the 332 IRGs were downloaded from TCGA. We defined a lncRNA with $(|\mathrm{R}|>0.7$ and $\mathrm{P}<0.05)$ expression levels between the lncRNAs and genes as IRLs. Thus, on the basis of Pearson correlation analysis, 52 lncRNAs were confirmed as IRLs $(|R|>0.7$ and $P<0.05)$. After univariate CPHRA, 4 IRLs were identified in the primary cohort $(P<0.05)$. Subsequent multivariate CPHRA further screened four IRLs (A1BG-AS1, AC004477.3, AC004585.1 and AC004854.2) as independent prognostic factors in the primary cohort. The VIFs ranged from 1.01 to 1.03 , indicating no collinearity among the predictors. The detailed characteristics of the four IRLs in the training dataset are listed in Table 2.
Table 1 Baseline characteristics of the included patients

\begin{tabular}{|c|c|c|c|}
\hline Variables & $\begin{array}{l}\text { Primary cohort } \\
\text { No. (\%) }\end{array}$ & $\begin{array}{l}\text { Validation cohort } \\
\text { No. }(\%)\end{array}$ & $P$ value \\
\hline No. of patients & 911 & 456 & \\
\hline Age (years) & $58(48,66)$ & $57(48,67)$ & 0.862 \\
\hline Tstage & & & 0.699 \\
\hline $\mathrm{T} 1$ & $248(27.2)$ & $125(27.4)$ & \\
\hline $\mathrm{T} 2$ & $529(58.1)$ & $254(55.7)$ & \\
\hline $\mathrm{T} 3$ & $110(12.1)$ & $65(14.3)$ & \\
\hline T4 & $24(2.6)$ & $12(2.6)$ & \\
\hline N stage & & & 0.266 \\
\hline No & $435(47.8)$ & $224(49.1)$ & \\
\hline $\mathrm{N} 1$ & $306(33.6)$ & $150(32.9)$ & \\
\hline N2 & $95(10.4)$ & $48(10.5)$ & \\
\hline N3 & $65(7.1)$ & $34(7.5)$ & \\
\hline $\mathrm{Nx}$ & $10(1.1)$ & $0(0)$ & \\
\hline TNM stage & & & 0.878 \\
\hline I & $161(17.7)$ & $82(18.0)$ & \\
\hline ॥ & $527(57.8)$ & $261(57.2)$ & \\
\hline III & $206(22.6)$ & $107(23.5)$ & \\
\hline Unknown & $17(1.9)$ & $6(1.3)$ & \\
\hline ER status & & & 0.723 \\
\hline Negative & $192(21.1)$ & $90(19.7)$ & \\
\hline Positive & $682(74.8)$ & $350(76.8)$ & \\
\hline Unknown & $37(4.1)$ & $16(3.5)$ & \\
\hline PR status & & & 0.920 \\
\hline Negative & $275(30.2)$ & $137(30.0)$ & \\
\hline Positive & $598(65.6)$ & $302(66.2)$ & \\
\hline Unknown & $38(4.2)$ & $17(3.7)$ & \\
\hline HER2 status & & & 0.265 \\
\hline Negative & $653(71.7)$ & $340(74.6)$ & \\
\hline Positive & $155(17.0)$ & $62(13.6)$ & \\
\hline Unknown & $103(11.3)$ & $54(11.8)$ & \\
\hline Molecular subtype & & & 0.495 \\
\hline $\mathrm{HR}+/ \mathrm{HER} 2-$ & $523(57.4)$ & $277(60.7)$ & \\
\hline $\mathrm{HR}+/ \mathrm{HER} 2+$ & $124(13.6)$ & $52(11.4)$ & \\
\hline HR-/HER2+ & $31(3.4)$ & $10(2.2)$ & \\
\hline TNBC & $129(14.2)$ & 62 (13.6) & \\
\hline Unknown & $104(11.4)$ & $55(12.1)$ & \\
\hline
\end{tabular}

TNM tumor-node-metastasis, $E R$ estrogen receptor, $P R$ progesterone receptor, HER2 human epithelial growth factor receptor 2

Table 2 The four-IRLs significantly associated with 5-year RFS in the primary cohort

\begin{tabular}{llllrr}
\hline Ensemble ID & Gene name & Genomic location & HR & P value & Coefficient \\
\hline ENSG00000268895 & A1BG-AS1 & chr 19:58,347,718-58,355,455 & 0.637 & 0.013 & -0.451 \\
ENSG00000278765 & AC004477.3 & chr 17: 48,066,704-48,067,293 & 1.341 & 0.007 & 0.294 \\
ENSG00000266088 & AC004585.1 & chr 17: 40,516,892-40,527,002 & 0.637 & 0.005 & -0.450 \\
ENSG00000272768 & AC004854.2 & chr 7: 44,884,953-44,886,393 & 1.873 & 0.001 & 0.627 \\
\hline
\end{tabular}

RFS relapse-free survival 
Table 3 Univariate and multivariate analyses in the primary cohort

\begin{tabular}{|c|c|c|c|c|}
\hline \multirow[t]{2}{*}{ Variables } & \multicolumn{2}{|l|}{ Univariate analysis } & \multicolumn{2}{|l|}{ Multivariate analysis } \\
\hline & HR $(95 \% \mathrm{Cl})$ & $P$ value & HR $(95 \% \mathrm{Cl})$ & $P$ value \\
\hline Age & $1.002(0.986-1.018)$ & 0.853 & & \\
\hline \multicolumn{5}{|l|}{ T stage } \\
\hline $\mathrm{T} 1$ & Referent & & & \\
\hline $\mathrm{T} 2$ & $1.631(0.980-2.714)$ & 0.060 & & \\
\hline T3 & $2.027(1.060-3.876)$ & 0.033 & & \\
\hline $\mathrm{T} 4$ & 5.748 (2.394-13.805) & $<0.001$ & & \\
\hline \multicolumn{5}{|l|}{ N stage } \\
\hline No & Referent & & & \\
\hline $\mathrm{N} 1$ & $1.611(1.004-2.585)$ & 0.048 & & \\
\hline N2 & $2.282(1.219-4.269)$ & 0.010 & & \\
\hline N3 & $4.651(2.425-8.921)$ & $<0.001$ & & \\
\hline $\mathrm{Nx}$ & 15.987 (6.600-38.722) & $<0.001$ & & \\
\hline \multicolumn{5}{|l|}{ TNM stage } \\
\hline I & Referent & & Referent & \\
\hline$\|$ & $1.641(0.850-3.167)$ & 0.140 & 1.589 (0.820-3.077 & 0.170 \\
\hline III & $3.589(1.824-7.061)$ & $<0.001$ & $4.015(2.010-8.020)$ & $<0.001$ \\
\hline Unknown & $13.559(5.415-33.951)$ & $<0.001$ & $12.054(4.623-31.430)$ & $<0.001$ \\
\hline \multicolumn{5}{|l|}{ ER status } \\
\hline Negative & Referent & & & \\
\hline Positive & $0.562(0.369-0.855)$ & 0.007 & & \\
\hline Unknown & $1.097(0.426-2.825)$ & 0.848 & & \\
\hline \multicolumn{5}{|l|}{ PR status } \\
\hline Negative & Referent & & & \\
\hline Positive & $0.550(0.369-0.821)$ & 0.003 & & \\
\hline Unknown & $1.120(0.442-2.838)$ & 0.811 & & \\
\hline \multicolumn{5}{|l|}{ Tumor subtype } \\
\hline HR+/HER2- & Referent & & Referent & \\
\hline $\mathrm{HR}+/ \mathrm{HER} 2+$ & $0.756(0.339-1.687)$ & 0.495 & $0.792(0.354-1.774)$ & 0.571 \\
\hline HR-/HER2+ & $1.744(0.624-4.872)$ & 0.289 & $0.924(0.320-2.672)$ & 0.884 \\
\hline TNBC & $2.038(1.204-3.450)$ & 0.008 & $2.318(1.359-3.955)$ & 0.002 \\
\hline Unknown & $2.591(1.572-4.269)$ & $<0.001$ & $1.922(1.153-3.204)$ & 0.012 \\
\hline Risk score & $2.718(1.903-3.884)$ & $<0.001$ & $2.975(2.028-4.364)$ & $<0.001$ \\
\hline
\end{tabular}

$C l$ confidence interval, $H R$ hazard ratios, $E R$ estrogen receptor, $P R$ progesterone receptor, $H E R 2$, human epithelial growth factor receptor 2

Italic values indicate statistical significance $(P<0.05)$

\section{Development of the IRL-based risk score and prognostic nomogram}

An IRL-based risk score formula was built on the basis of IRL expression levels and their estimated regression coefficients in the multivariate CPHRA, where IRL-based risk score $=-0.451 \times$ expression $_{\mathrm{A} 1 \mathrm{BG}-}$

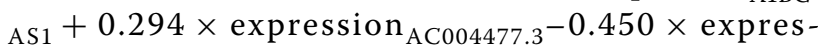
sion $_{\mathrm{AC} 004585.1}+0.627 \times$ expression $_{\mathrm{AC} 004854.2}$. Based on univariate and multivariate CPHRA (Table 3), three prognostic variables (IRL-based risk score, tumor subtype, and TNM stage) were selected as independent indicators to build an IRL-based model. The novel IRL-based nomogram is illustrated in Fig. 1.

Points
Risk score
TNM stage



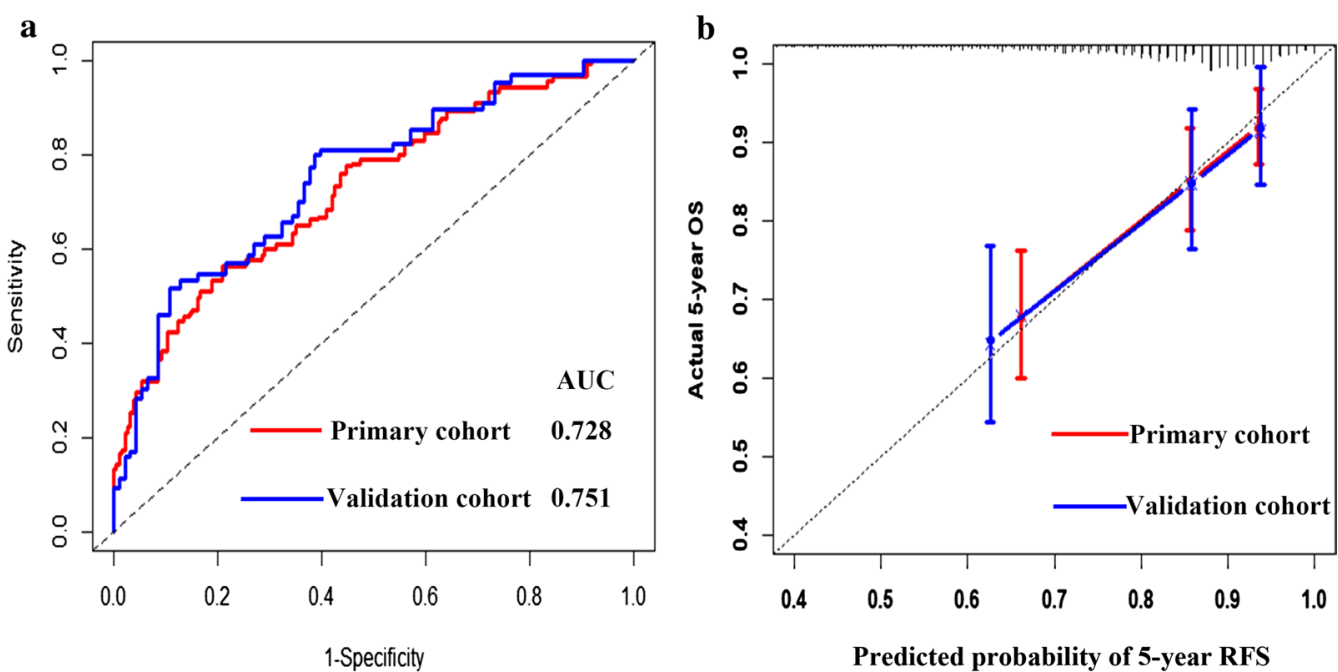

Predicted probability of 5-year RFS

Fig. 2 a Time-dependent receiver operating characteristic (ROC) curves at 5-years based on the immune-related IncRNAs model in the primary cohort and validation cohort. b Calibration curves of the immune-related IncRNAs model in the primary cohort and validation cohort

\section{Evaluation of the IRL-based prognostic nomogram}

The AUCs of the IRL-based nomogram were 0.728 (95\% CI: $0.658-0.797$ ) and 0.751 (95\% CI: 0.656-0.846) in the primary and validation datasets, respectively, showing that this model had good predictive performance
(Fig. 2a). Calibration plots revealed ideal agreement between the IRL-based nomogram-predicted likelihoods and the actual observations of 5-year RFS, indicating that this tool had high calibration ability (Fig. 2b). In addition, compared with the predictive accuracy between the
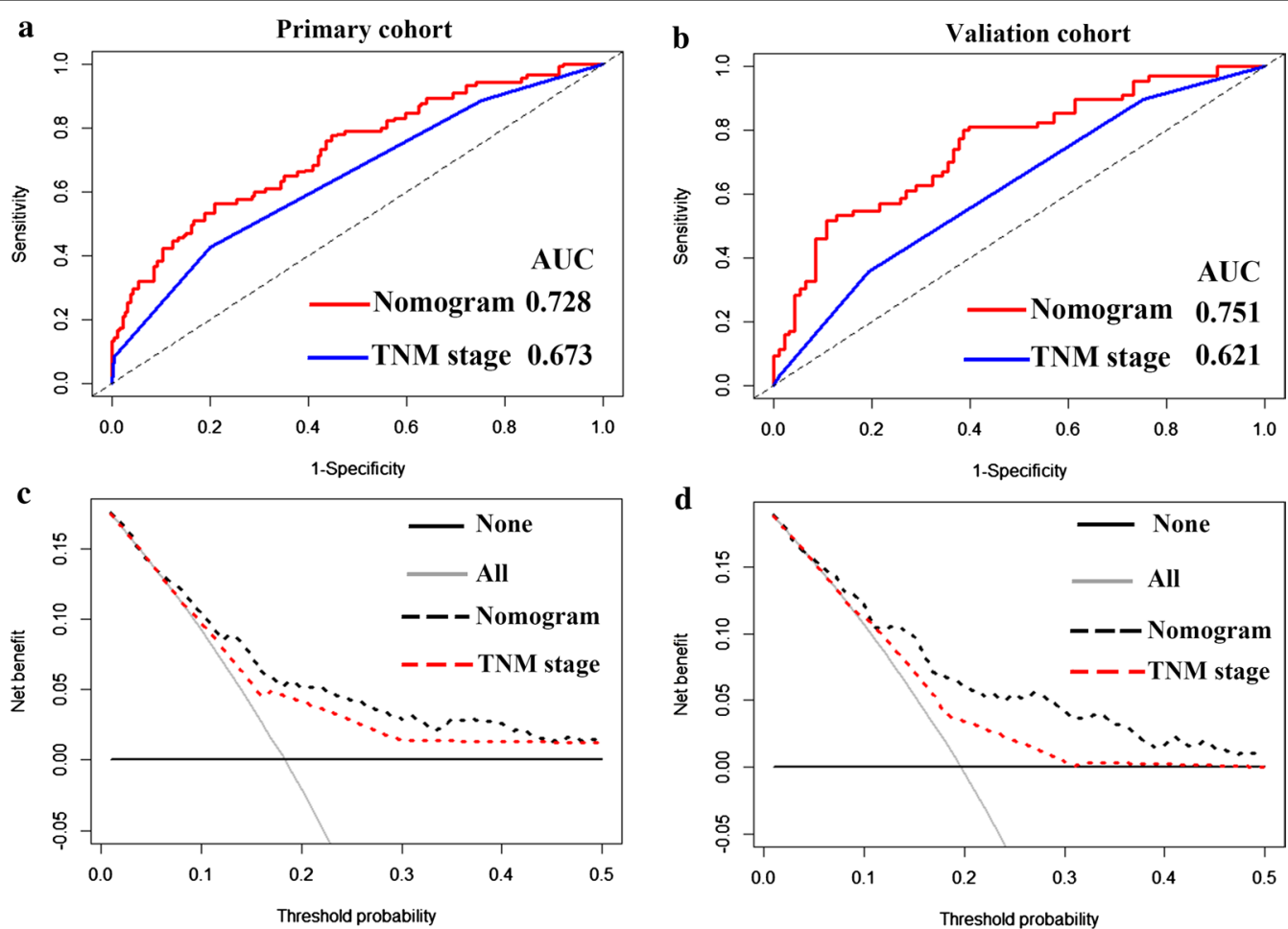

Fig. 3 Comparisons of the predictive accuracy between the immune-related IncRNAs model and TNM stage using time-dependent ROC curves in the primary cohort (a) and validation cohort (b). Comparisons of the clinical utility between the immune-related IncRNAs model and TNM stage using decision curve in the primary cohort (c) and validation cohort (d) 
IRL-based model and TMN stage, the time-dependent ROC curve suggested that the IRL-based nomogram outperformed the TNM stage for both the primary and validation cohorts $(P<0.05$, Fig. $3 \mathrm{a}, \mathrm{b})$. Moreover, DCA indicated that the IRL-based tool added more net benefit than did the TNM stage for the primary and validation datasets; therefore, this nomogram showed superior clinical usefulness (Fig. 3c, d). On the basis of the IRLbased model, patients in the primary and validation cohorts were stratified into low- or high-risk groups using the median nomogram score as the cutoff point. The distribution characteristics of the IRL-based model score, RFS, and RFS status are shown in Fig. 4, indicating that patients with lower nomogram scores had better 5-year RFS than those with higher nomogram scores $(P<0.001)$. Subgroup analyses demonstrated that the IRL-based model had good risk stratification ability for T1 $(P<0.0001)$, T2 $(P<0.0001)$, T3 $(P=0.0067)$, N0 $(P=0.029)$, N1 $(P<0.0001)$, N2 $(P=0.013)$, HR $+/$ HER2$(P=0.00033), \quad$ HR-/HER $2+(P=0.038), \quad$ and TNBC $(P<0.0001)$ (Fig. 5).

\section{Functional enrichment analysis of the IRL signature}

To further explore the potential functional roles of the four IRLs, significantly associated IRGs (Pearson coefficient $>0.7$ and $P<0.05)$ were included in pathway enrichment via Metascape. The top 20 highly enriched pathways are displayed in Fig. 6. The IRGs of the four IRLs clustered most significantly in lymphocyte activation, cytokine-mediated signaling pathway, regulation of cytokine production, negative regulation of immune system process, regulation of immune effector process, alpha-beta $\mathrm{T}$ cell activation, leukocyte migration, antigen receptor-mediated signaling pathway, B cell activation, TCR pathway, response to bacterium, $\mathrm{T}$ cell costimulation, regulation of peptidyl-tyrosine phosphorylation, calciummediated signaling, myeloid leukocyte activation, positive regulation of defense response, lymphocyte migration, interleukin-10 signaling, positive regulation of cytosolic calcium ion concentration, and regulation of interferon-gamma production categories. Moreover, according to LncRRIsearch, a web tool used for comprehensive prediction of lncRNA-mRNA interactions [43], the predicted targets of A1BG-AS1 are FKBP10, NFYC, UBR4, CEACAM19, MT-ND4, PKD1P6, and RNA28S5. The predicted target of AC004477.3 is SEH1L.
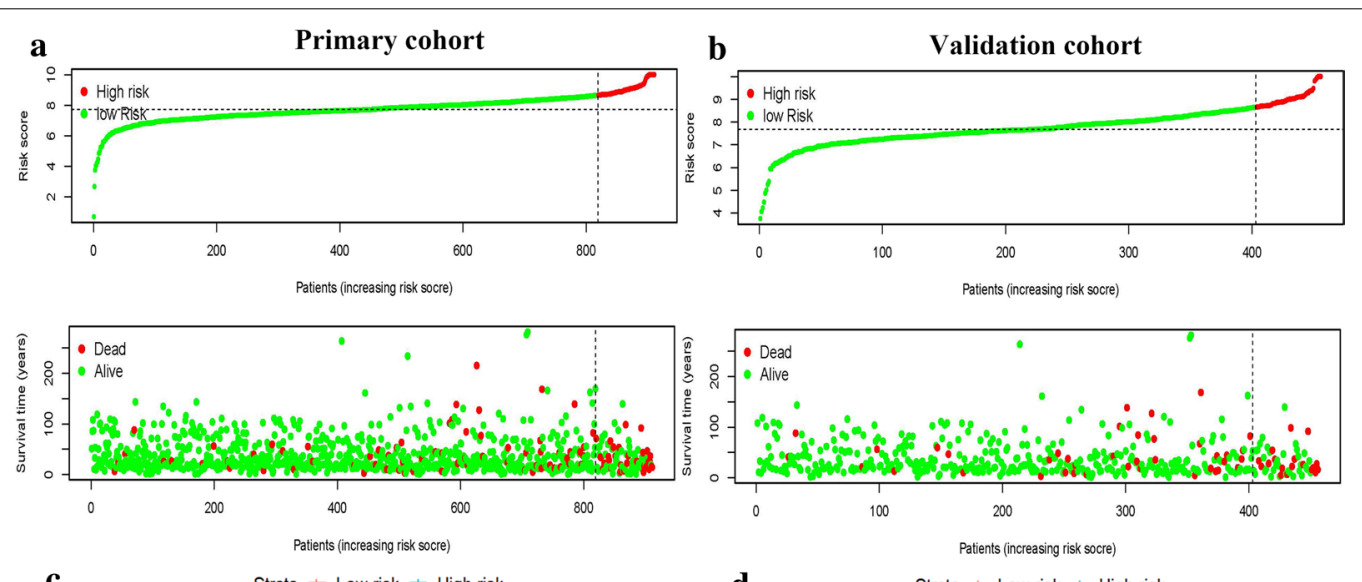

C

Strata + Low-risk + High-risk

d Patients (increasing risk socre)
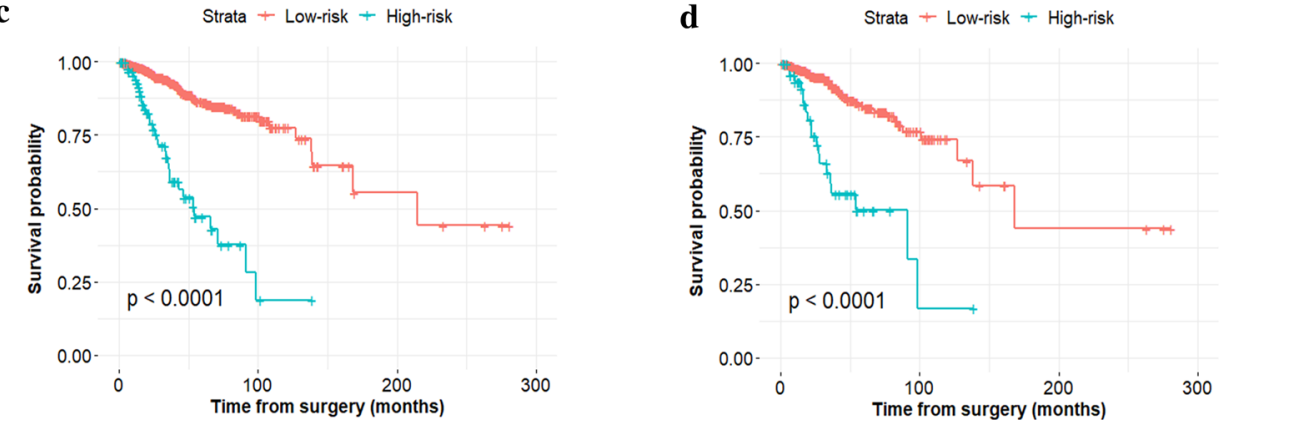

Fig. 4 The distribution of immune-related IncRNAs model score, RFS, and RFS status in the primary cohort (a) and validation cohort (b). The dotted line indicates the optimal cutoff value of the model score to divide patients into the low- and high-risk set. Kaplan-Meier curves of the low-and high-risk patients based on the immune-related IncRNAs model in the primary cohort (c) and validation cohort (d) 


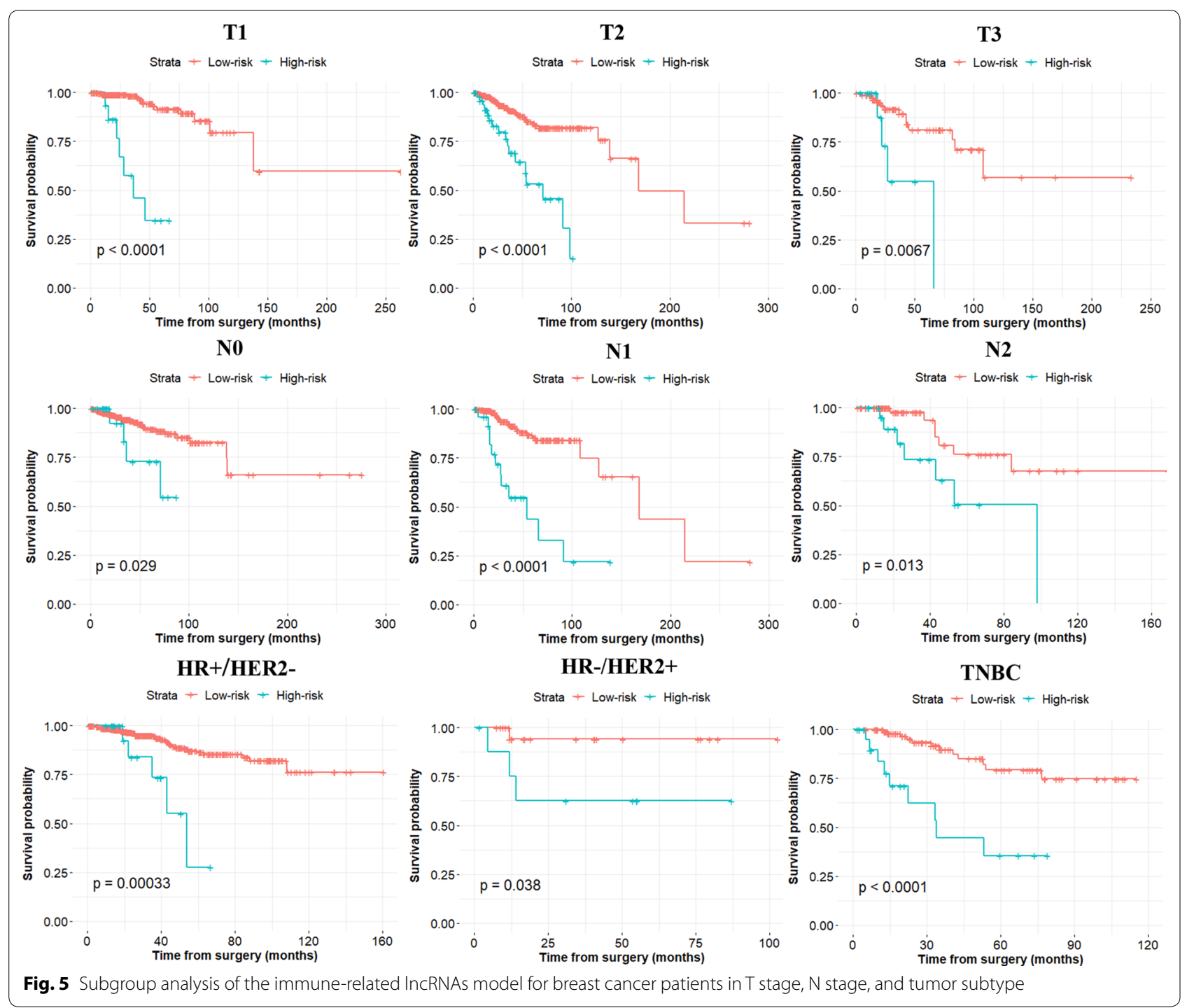

\section{Discussion}

With the increasing development of clinical management and comprehensive treatment of $\mathrm{BC}$, prognosis of the survival of these patients has greatly improved. Nevertheless, many $\mathrm{BC}$ patients experience tumor recurrence [44]. High-throughput biological technologies have provided a platform for exploring the molecular characteristics of different tumors [45]. Many lncRNAs have been used to predict $\mathrm{BC}$ prognosis $[44,46,47]$, though few IRL signatures have been identified as improving $\mathrm{BC}$ survival prediction. Consequently, it is necessary to single out prognostic IRLs integrating TNM stage to predict 5-year RFS of BC patients. In this study, with the combination of four IRLs, TNM stage and tumor subtype, we efficiently constructed and validated a novel IRL-based nomogram to accurately predict 5-year RFS in BC patients.
In addition, time-dependent $\mathrm{ROC}$ analysis revealed that the IRL-based nomogram had more favorable prognostic accuracy than did TNM stage. Moreover, the DCA indicated that the IRL-based model had better clinical application than TNM stage; our IRL-based tool also exhibited good risk stratification ability to significantly categorize $\mathrm{BC}$ patients into high- and low-risk groups.

To date, several prognostic tools have been established to predict survival in BC patients [44, 46-55]. Yao et al. identified five lncRNAs as vital prognostic factors to evaluate $\mathrm{BC}$ prognosis [46]. However, these models ignored the prognostic value of IRLs for BC patients. Recent studies have suggested that the immune response plays a critical role in cancer progression and recurrence, and accumulating evidence has revealed that IRLs harbor vital predictive value for survival prognosis. For example, 


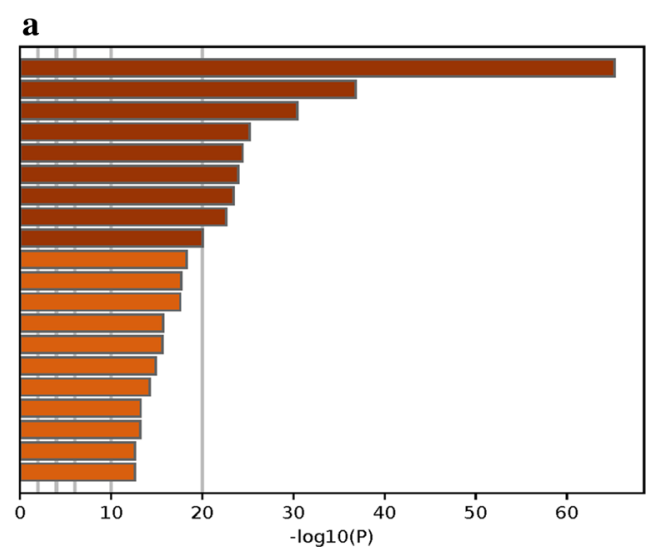

0.0046649: lymphocyte activation

GO:0019221: cytokine-mediated signaling pathway

GO:0001817: regulation of cytokine production

GO:0002683: negative regulation of immune system process

GO:0002697: regulation of immune effector process

GO:0046631: alpha-beta T cell activation

GO:0050900: leukocyte migration

GO:0050900: leukocyte migration

GO:0042113: B cell activation

M34: PID TCR PATHWAY

GO:0009617: response to bacterium

GO:0031295: T cell costimulation

GO:0050730: regulation of peptidyl-tyrosine phosphorylation

GO:0019722: calcium-mediated signaling

GO:0002274: myeloid leukocyte activation

GO:0031349: positive regulation of defer

GO:0072676: lymphocyte migration
R-HSA-6783783: Interleukin-10 signaling

GO:0007204: positive regulation of cytosolic calcium ion concentration

GO:0032649: regulation of interferon-gamma production

b

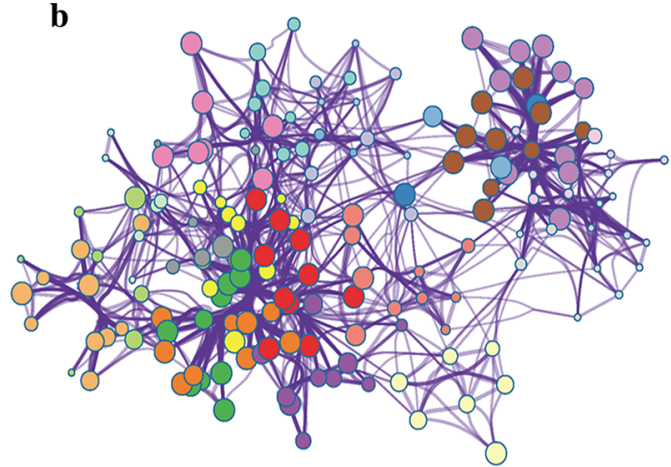

Elymphocyte activation

cytokine-mediated signaling pathway

kine production

negative regulation of immune system process
regulation of immune effector process

alpha-beta T cell activation

leukocyte migration

antigen receptor-mediated signaling pathway

B cell activation

response to bacterium

T cell costimulation

regulation of peptidyl-tyrosine phosphorylation

calcium-mediated signaling

myeloid leukocyte activatio

positive regulation of defense response

Iymphocyte migration

positive regulation of cytosolic calcium ion concentra

regulation of interferon-gamma production

Fig. 6 Gene enrichment analysis of the immune-related IncRNAs signature in the Metascape database. a The 20 enrichment terms. $\mathbf{b}$ The network of the enrichment terms

Zhang et al. found that si-lncMALAT1 both suppressed osteosarcoma progression and resulted in self-destruction of cells deep within the tumor. This would enable clearance of osteosarcoma cells from the body by the immune system. Therefore, IncMALAT1 was individually correlated with immune system activity and overall survival [56]. LncRNAs cannot encode proteins but do regulate gene expression at different levels, including through epigenetic, transcriptional or posttranscriptional regulation [57]. In the past decade, this new type of gene regulator, namely, lncRNA, has been associated with tumor development, progression, and prognosis, and many lncRNAs have been found to act as prognostic indicators in various tumors. Moreover, lncRNAs can regulate the immune microenvironment of $B C$ [58]. IRLs can be applied to characterize the infiltration of immune cells in tumors. In our study, the IRL signature developed had a better prognostic value than the IRG signature. Thus, IRLs should be integrated into nomograms to improve prognosis prediction in BC. The survival prediction based on the TNM staging system is not yet satisfactory in clinical practice [59], though it is universally acknowledged that the TNM staging system is beneficial to achieve optimal treatment choice for BC patients.
The TNM staging system is based on tumor size, lymph node status and metastasis [60]. Nonetheless, malignant behavior in $\mathrm{BC}$ is mainly determined by the molecular characteristics. Thus, incorporating the TNM staging system and molecular biomarkers is indispensable for developing an accurate tool for prognostic assessment in BC patients [61].

Some limitations should be noted in the present study. First, TCGA does not include postoperative treatment information (chemotherapy and hormone therapy). Therefore, we are unable to screen low-risk patients to tailor adjuvant treatment. Second, the molecular mechanism of the IRLs should be further explored by additional experiments. Third, our established model should be verified by prospective, large-scale multicenter datasets.

\section{Conclusions}

In summary, we identified IRLs signatures significantly associated with the 5-year RFS of BC and efficiently constructed a novel prognostic model for 5-year RFS prediction by incorporating four IRLs, TNM stage and tumor subtype. Our model can improve the predictive power of the AJCC TNM staging system and confirm high-risk 


\section{patients with tumor recurrence to receive appropriate treatment.}

\begin{abstract}
Abbreviations
IRLs: Immune-related IncRNAs; BC: Breast cancer; TCGA: The Cancer Genome Atlas; RFS: Recurrence-free survival; DCA: Decision curve analysis; AJCC: American Joint Committee on Cancer; TNM: Tumor-node-metastasis; IncRNAs: Long non-coding RNAs; ER: Estrogen receptor; PR: Progesterone receptor; HER2: Human epithelial growth factor receptor 2; GDPH: Guangdong Provincial People's Hospital; IRGs: Immune-related genes; MSDB: Molecular Signatures Database; LASSO: Least absolute shrinkage and selection operator; CPHRA: Cox proportional hazards regression analysis; AUC: Area under the curve; ROC: Receiver operating characteristic.
\end{abstract}

\section{Acknowledgements \\ Not applicable.}

\section{Authors' contributions}

$J G L, B C$ and NL conceived and designed this study; GCZ provided the clinical specimens; JGL and HM acquired the data; XRL analyzed the data and results; $\mathrm{JGL}$ and $\mathrm{BC}$ wrote the manuscript; NL improved and revised the manuscript. All authors read and approved the final manuscript.

\section{Funding}

This study was supported by the Doctor Launch Fund of Guangdong Provincial People's hospital (grant number 2020bq11), the National Natural Science Foundation of China (Grants 82002928; 81902828; 81602645; 81071851; 81001189), National Natural Science Foundation of Guangdong Province (Grant 2016A030313768; 2018A030313292), Medical Scientific Research Foundation of Guangdong Province (B2019039, Bo Chen), Research Funds from Guangzhou Municipal Science and Technology Project (201707010418; 201804010430).

\section{Availability of data and materials}

All data was obtained from The Cancer Genome Atlas (TCGA, https://tcgad atanci.nih.gov/tcga/).

\section{Ethics approval and consent to participate}

Not applicable.

\section{Consent for publication}

Not applicable.

\section{Competing interests}

The authors declare that they have no competing interests.

Received: 16 May 2020 Accepted: 24 October 2020

Published online: 07 November 2020

\section{References}

1. Yap Y-S, Lu Y-S, Tamura K, Lee JE, Ko EY, Park YH, Cao AY, Lin C-H, Toi M, Wu J, Lee S-C. Insights Into Breast Cancer in the East vs the West. JAMA Oncology. 2019;12:63.

2. Harbeck N, Penault-Llorca F, Cortes J, Gnant M, Houssami N, Poortmans P, Ruddy K. Tsang J. Cardoso F: Breast cancer. Nature Reviews Disease Primers; 2019. p. 5 .

3. Siegel RL, Miller KD, Jemal A. Cancer statistics, 2020. CA Cancer J Clin. 2020;70:7-30.

4. Lai J, Chen B, Zhang G, Wang Y, Mok H, Wen L, Pan Z, Su F, Liao N. Identification of a novel microRNA recurrence-related signature and risk stratification system in breast cancer. Aging (Albany NY). 2019;11:7525-36

5. Lai J, Wang H, Pan Z, Su F. A novel six-microRNA-based model to improve prognosis prediction of breast cancer. Aging (Albany NY). 2019;11:649-62.

6. Lai J, Pan Z, Chen P, Ye G, Chen K, Su F. Development and validation of a nomogram incorporating axillary lymph node ratio to predict survival in node-positive breast cancer patients after neoadjuvant chemotherapy. Jpn J Clin Oncol. 2018;49:22-8.

7. Lai J, Wang H, Peng J, Chen P, Pan Z. Establishment and external validation of a prognostic model for predicting disease-free survival and risk stratification in breast cancer patients treated with neoadjuvant chemotherapy. Cancer Manag Res. 2018;10:2347-56.

8. Zhu X, Tian X, Sun T, Yu C, Cao Y, Yan T, Shen C, Lin Y, Fang JY, Hong J, Chen H. GeneExpressScore signature: a robust prognostic and predictive classifier in gastric cancer. Mol Oncol. 2018;33:459.

9. Wen J, Wang G, Xie X, Lin G, Yang H, Luo K, Liu Q, Ling Y, Xie X, Lin P, et al. Prognostic Value of a Four-miRNA signature in patients with lymph node positive locoregional esophageal squamous cell carcinoma undergoing complete surgical resection. Ann Surg. 2019;26:1.

10. Mitra AP, Lam LL, Ghadessi M, Erho N, Vergara IA, Alshalalfa M, Buerki C, Haddad Z, Sierocinski T, Triche TJ, et al. Discovery and validation of novel expression signature for postcystectomy recurrence in high-risk bladder cancer. J Natl Cancer Inst. 2014;22:106.

11. Zhu X, Tian X, Yu C, Shen C, Yan T, Hong J, Wang Z, Fang JY, Chen H. A long non-coding RNA signature to improve prognosis prediction of gastric cancer. Mol Cancer. 2016;15:60.

12. Qu L, Wang Z, Chen Q, Li Y, He H, Hsieh JJ, Xue S, Wu Z, Liu B, Tang H, et al. Prognostic value of a long non-coding RNA signature in localized clear cell renal cell carcinoma. Eur Urol. 2018;32:98.

13. Zhang J-X, Song W, Chen Z-H, Wei J-H, Liao Y-J, Lei J, Hu M, Chen G-Z, Liao $B$, Lu J, et al. Prognostic and predictive value of a microRNA signature in stage II colon cancer: a microRNA expression analysis. Lancet Oncol. 2013;14:1295-306

14. Tang X-R, Li Y-Q, Liang S-B, Jiang W, Liu F, Ge W-X, Tang L-L, Mao Y-P, He $\mathrm{Q}-\mathrm{M}$, Yang $X-J$, et al. Development and validation of a gene expressionbased signature to predict distant metastasis in locoregionally advanced nasopharyngeal carcinoma: a retrospective, multicentre, cohort study. The Lancet Oncology. 2018;65:523.

15. Zhou W, Gong J, Chen Y, Chen J, Zhuang Q, Cao J, Mei Z, Hu B. Long noncoding RNA LINC00899 suppresses breast cancer progression by inhibiting miR-425. Aging (Albany NY). 2019;11:425.

16. Zhang N, Zeng X, Sun C, Guo H, Wang T, Wei L, Zhang Y, Zhao J, Ma X. LncRNA LINC00963 Promotes Tumorigenesis and Radioresistance in Breast Cancer by Sponging miR-324-3p and Inducing ACK1 Expression. Mol Ther Nucleic Acids. 2019;18:871-81.

17. Zhang G, Xu Y, Zou C, Tang Y, Lu J, Gong Z, Ma G, Zhang W, Jiang P. Long noncoding RNA ARHGAP27P1 inhibits gastric cancer cell proliferation and cell cycle progression through epigenetically regulating p15 and p16. Aging (Albany NY). 2019;11:9090-110.

18. Yang Z, An Y, Wang N, Dong X, Kang H. LINC02595 promotes tumor progression in colorectal cancer by inhibiting miR-203b-3p activity and facilitating BCL2L1 expression. J Cell Physiol. 2020;28:65.

19. Tan BS, Yang MC, Singh S, Chou YC, Chen HY, Wang MY, Wang YC, Chen RH. LnCRNA NORAD is repressed by the YAP pathway and suppresses lung and breast cancer metastasis by sequestering S100P. Oncogene. 2019;38:5612-26

20. Shen SN, Li K, Liu Y, Yang CL, He CY, Wang HR. Silencing IncRNAs PVT1 Upregulates miR-145 and confers inhibitory effects on viability, invasion, and migration in EC. Mol Ther Nucleic Acids. 2019;19:668-82.

21. Ren X, Chen C, Luo Y, Liu M, Li Y, Zheng S, Ye H, Fu Z, Li M, Li Z, Chen R. IncRNA-PLACT1 sustains activation of NF-kappaB pathway through a positive feedback loop with IkappaBalpha/E2F1 axis in pancreatic cancer. Mol Cancer. 2020;19:35.

22. Ji W, Diao YL, Qiu YR, Ge J, Cao XC, Yu Y. LINC00665 promotes breast cancer progression through regulation of the miR-379-5p/LIN28B axis. Cell Death Dis. 2020;11:16.

23. Guan Z, Wang Y, Wang Y, Liu X, Wang Y, Zhang W, Chi X, Dong Y, Liu X, Shao S, Zhan Q. Long non-coding RNA LOC100133669 promotes cell proliferation in oesophageal squamous cell carcinoma. Cell Prolif. 2020;2020:e12750.

24. Dong HT, Liu Q, Zhao T, Yao F, Xu Y, Chen B, Wu Y, Zheng X, Jin F, Li J, Xing P. Long non-coding RNA LOXL1-AS1 drives breast cancer invasion and metastasis by antagonizing miR-708-5p expression and activity. Mol Ther Nucleic Acids. 2019;19:696-705.

25. Fu J, Dong G, Shi H, Zhang J, Ning Z, Bao X, Liu C, Hu J, Liu M, Xiong B. LncRNA MIR503HG inhibits cell migration and invasion via miR-103/ OLFM4 axis in triple negative breast cancer. J Cell Mol Med. 2019;2019:38. 
26. Pei $X$, Wang $X$, Li H. LncRNA SNHG1 regulates the differentiation of Treg cells and affects the immune escape of breast cancer via regulating miR448/IDO. Int J Biol Macromol. 2018;118:24-30.

27. Chen J, Wang S, Jia S, Ding G, Jiang G, Cao L. Integrated analysis of long non-coding RNA and mRNA expression profile in pancreatic cancer derived exosomes treated dendritic cells by microarray analysis. J Cancer. 2018;9:21-31.

28. Zhou M, Zhao H, Wang Z, Cheng L, Yang L, Shi H, Yang H, Sun J. Identification and validation of potential prognostic IncRNA biomarkers for predicting survival in patients with multiple myeloma. J Exp Clin Cancer Res. 2015;34:102.

29. Zhou M, Zhang Z, Zhao H, Bao S, Cheng L, Sun J. An immune-Related Six-IncRNA signature to improve prognosis prediction of glioblastoma multiforme. Mol Neurobiol. 2018;55:3684-97.

30. Zhou H, Zhang H, Chen J, Cao J, Liu L, Guo C, Huang G, Zeng D. A sevenlong noncoding RNA signature predicts relapse in patients with earlystage lung adenocarcinoma. J Cell Biochem. 2019;12:315.

31. Zhang C, Li Z, Hu J, Qi F, Li X, Luo J. Identification of five long noncoding RNAs signature and risk score for prognosis of bladder urothelial carcinoma. Journal of Cellular Biochemistry. 2019. https://doi.org/10.1002/ jcb.29330.

32. Zhang B, Wang H, Guo Z, Zhang X. Prediction of head and neck squamous cell carcinoma survival based on the expression of 15 IncRNAs. J Cell Physiol. 2019;36:15.

33. Barbie DA, Tamayo P, Boehm JS, Kim SY, Moody SE, Dunn IF, Schinzel AC, Sandy P, Meylan E, Scholl C, et al. Systematic RNA interference reveals that oncogenic KRAS-driven cancers require TBK1. Nature. 2009;462:108-12.

34. Wang W, Zhao Z, Yang F, Wang H, Wu F, Liang T, Yan X, Li J, Lan Q, Wang J, Zhao J. An immune-related IncRNA signature for patients with anaplastic gliomas. J Neurooncol. 2018;136:263-71.

35. Lossos IS, Czerwinski DK, Alizadeh AA, Wechser MA, Tibshirani R, Botstein D, Levy R. Prediction of survival in diffuse large-B-cell lymphoma based on the expression of six genes. N Engl J Med. 2004;350:1828-37.

36. Vickers AJ, Van Calster B, Steyerberg EW. Net benefit approaches to the evaluation of prediction models, molecular markers, and diagnostic tests. BMJ. 2016:352:i6.

37. Vickers AJ, Elkin EB. Decision curve analysis: a novel method for evaluating prediction models. Med Decis Making. 2006;26:565-74.

38. Kastrinos F, Ojha RP, Leenen C, Alvero C, Mercado RC, Balmana J, Valenzuela I, Balaguer F, Green R, Lindor NM, et al. Comparison of prediction models for lynch syndrome among individuals with colorectal cancer. J Natl Cancer Inst. 2016;22:108.

39. Huang YQ, Liang CH, He L, Tian J, Liang CS, Chen X, Ma ZL, Liu ZY. Development and validation of a radiomics nomogram for preoperative prediction of lymph node metastasis in colorectal cancer. J Clin Oncol. 2016;34:2157-64

40. Han SS, Rivera GA, Tammemagi MC, Plevritis SK, Gomez SL, Cheng I, Wakelee HA. Risk stratification for second primary lung cancer. J Clin Oncol. 2017:35:2893-9.

41. Kerr KF, Brown MD, Zhu K, Janes H. Assessing the clinical impact of risk prediction models with decision curves: guidance for correct interpretation and appropriate use. J Clin Oncol. 2016;34:2534-40.

42. Jassal B, Matthews L, Viteri G, Gong C, Lorente P, Fabregat A, Sidiropoulos K, Cook J, Gillespie M, Haw R, et al. The reactome pathway knowledgebase. Nucleic Acids Res. 2020:48:D498-d503.

43. Fukunaga T, Iwakiri J, Ono Y, Hamada M. LncRRIsearch: A web server for IncRNA-RNA interaction prediction integrated with tissue-specific expression and subcellular localization data. Front Genet. 2019;10:462.
44. Tang J, Ren J, Cui Q, Zhang D, Kong D, Liao X, Lu M, Gong Y, Wu G. A prognostic 10-IncRNA expression signature for predicting the risk of tumour recurrence in breast cancer patients. J Cell Mol Med. 2019;2:35.

45. Lai J, Chen B, Mok H, Zhang G, Ren C, Liao N. Comprehensive analysis of autophagy-related prognostic genes in breast cancer. J Cell Mol Med. 2020;24:9145-53

46. Yao Y, Zhang T, Qi L, Zhou C, Wei J, Feng F, Liu R, Sun C. Integrated analysis of co-expression and ceRNA network identifies five IncRNAs as prognostic markers for breast cancer. J Cell Mol Med. 2019;20:98.

47. Meng J, Li P, Zhang Q, Yang Z, Fu S. A four-long non-coding RNA signature in predicting breast cancer survival. J Exp Clin Cancer Res. 2014;33:84.

48. Wang K, Liao C, Zhong Q, Dong H, Zhang T, Jin R. CeNETs analysis reveals the prognostic value of a signature integration from five IncRNAs in breast cancer. J Cell Biochem. 2019;56:33.

49. Tian T, Gong Z, Wang M, Hao R, Lin S, Liu K, Guan F, Xu P, Deng Y, Song D, et al. Identification of long non-coding RNA signatures in triple-negative breast cancer. Cancer Cell Int. 2018;18:103.

50. Tang J, Cui Q, Zhang D, Kong D, Liao X, Ren J, Gong Y, Wu G. A prognostic eight-IncRNA expression signature in predicting recurrence of ER-positive breast cancer receiving endocrine therapy. J Cell Physiol. 2019;235:4746.

51. Sun M, Wu D, Zhou K, Li H, Gong X, Wei Q, Du M, Lei P, Zha J, Zhu H, et al. An eight-IncRNA signature predicts survival of breast cancer patients: a comprehensive study based on weighted gene co-expression network analysis and competing endogenous RNA network. Breast Cancer Res Treat. 2019;175:59.

52. Li J, Wang W, Xia P, Wan L, Zhang L, Yu L, Wang L, Chen X, Xiao Y, Xu C. Identification of a five-IncRNA signature for predicting the risk of tumor recurrence in patients with breast cancer. Int J Cancer. 2018;143:2150-60.

53. Li J, Gao C, Liu C, Zhou C, Ma X, Li H, Li J, Wang X, Qi L, Yao Y, et al. Four IncRNAs associated with breast cancer prognosis identified by coexpression network analysis. J Cell Physiol 2019.

54. He Y, Li X, Meng Y, Fu S, Cui Y, Shi Y, Du H. A prognostic 11 long noncoding RNA expression signature for breast invasive carcinoma. J Cell Biochem 2019.

55. Fan CN, Ma L, Liu N. Comprehensive analysis of novel three-long noncoding RNA signatures as a diagnostic and prognostic biomarkers of human triple-negative breast cancer. J Cell Biochem 2018.

56. Zhang ZC, Tang C, Dong Y, Zhang J, Yuan T, Li XL. Targeting LncRNAMALAT1 suppresses the progression of osteosarcoma by altering the expression and localization of beta-catenin. J Cancer. 2018;9:71-80.

57. Cai P, Otten A, Cheng B, Ishii M, Zhang W, Huang B, Qu K, Sun B. PRANCRA genome-wide long noncoding RNA CRISPRi screen identifies as a novel regulator of epidermal homeostasis. Genome Res. 2020;30:22-34.

58. Li Z, Li Y, Wang X, Yang Q. Identification of a Six-Immune-Related Long Non-coding RNA Signature for Predicting Survival and Immune Infiltrating Status in Breast Cancer. Front Genet. 2020;11:680.

59. Pennathur A, Luketich JD. Resection for esophageal cancer: strategies for optimal management. Ann Thorac Surg. 2008;85:S751-756.

60. lasonos A, Schrag D, Raj GV, Panageas KS. How to build and interpret a nomogram for cancer prognosis. J CLIN ONCOL. 2008;26:1364-70.

61. Balachandran VP, Gonen M, Smith JJ, DeMatteo RP. Nomograms in oncology: more than meets the eye. Lancet Oncol. 2015;16:e173-80.

\section{Publisher's Note}

Springer Nature remains neutral with regard to jurisdictional claims in published maps and institutional affiliations. 